\title{
A systematic review on the prevalence, pathophysiology, diagnosis, management and treatment of gout (2007-2018)
}

\author{
Ashiq Kanwal 1,2*, Latif Abida ${ }^{1}$, Ashiq Sana ${ }^{3}$, Sundus Ahlam ${ }^{2}$ \\ ${ }^{1}$ University College of Pharmacy, University of the Punjab Lahore, Pakistan. \\ 2 Lahore Pharmacy College, Lahore Medical and Dental College Lahore, Pakistan. \\ ${ }^{3}$ Department of Microbiology and Molecular Genetics, University of the Punjab Lahore, Pakistan.
}

Publication history: Received on 09 August 2018; revised on 24 September 2018; accepted on 04 October 2018

Article DOI: https://doi.org/10.30574/gscbps.2018.5.1.0077

\begin{abstract}
Gout is a type of inflammatory arthritis with drastically increasing incidence since last twenty years. Increased serum uric acid levels and deposition of urate crystals in joints and soft tissues are known to stir-up the symptoms of gout. Patient's quality of life (Qol) suffers markedly due to inflammation, pain, restricted mobility, health economic burden and suboptimal therapeutics associated with gout. Related articles published between 2007 and 2018 were reviewed with the help of different databases including PubMed, Springer link, Medline, Google scholar and Science direct. To ensure the accuracy and credibility of data, articles published only in indexed journals were considered. Gout is a common metabolic disorder that is provoked by increased level of serum uric acid. Presence of other comorbidities i.e. hypertension, diabetes and cardiac diseases precipitate the rate of mortality. Number of gout patients is shown to increase in developed countries whereas developing countries remain at high risk category. The enormous increase in gout is due to chronicity, lack of awareness among patients, poor diagnosis, suboptimal treatment and economic burden. Risk factors associated with gout are well identified and pathophysiology is well-established hence, measures can be taken to improve diagnosis and to ensure cost effective options for the treatment. To curtail the global health burden and to improve patient quality of life there is need: to reduce exposure to risk factors, to promote awareness, to institute robust prophylactic measures, to modify lifestyle, to establish signs for early diagnosis and to develop optimized treatment strategies and policies.
\end{abstract}

Keywords: Gout; Inflammation; Arthritis; Urate crystals; Hyperuricemia

\section{Introduction}

Gout is a common chronic inflammatory condition of joints recognized since ages [1]. Previously, gout was considered as a benign disease which was known to be provoked by either alcohol consumption or overeating [2]. Later, studies confirmed that it is a metabolic disorder which results due to the deposition of urate crystals in kidney, joints, skin and various other tissues [3]. It is also known as "men disease" because its occurrence is quite greater in males than females [4]. Prevalence of gout in men is four times greater than in females which is probably due to the presence of estradiol in females that inhibits the synthesis of urate crystals [5]. Gout is associated with multiple comorbidities including diabetes [4], hypertension, stroke and myocardial infarction being the most common of all [6]. Presence of other comorbidities raise the mortality rate and decline overall life expectancy [7]. Raised serum uric acid (about more than $6.8 \mathrm{mg} / \mathrm{dl}$ ) is known as hyperuricemia which is the sign for the gout manifestation [8].

A surge in serum uric acid generates urate crystals which is followed by the formation of renal stones and development of tophi that subsequently progress towards the gouty arthritis [9]. The disease spectrum extends from subclinical

\footnotetext{
${ }^{*}$ Corresponding author

E-mail address: kanwal.ashiq@Imdc.edu.pk
} 
hyperuricemia to acute gouty arthritis then to chronic tophaceous gout, which if persists for longer time eventually leads to more severe stage called chronic arthopathic gout [10]. Prevalence of gout is quite common among developed countries whereas, in emerging countries occurrence of disease is noticeably high. Increased and chronic consumption of meat, alcohol, sugar and protein rich diet are the key factors for the initiation and aggravation of gout [11]. Acute pain, subcutaneous tophi and persistence of low grade inflammation for longer time cause deformation of joints, restricted mobility and permanent disability that negatively influence patient's Qol [12]. Deposition of uric acid crystal may often damage kidneys and the condition may even progress to chronic nephritis [13].

Globally, the economic burden of disease has drastically increased [14] due to the suboptimal disease care, poor diagnosis [15] lack of awareness, communication gap among patients and health care professionals, little understanding of disease and its treatment and inaccessibility of medicines [16]. Increased hospitalization of gout patients is also a major health concern because of its direct influence on the cost of treatment [17]. Due to multiple comorbidities, it is difficult to estimate exact economic burden exerted by gout. However, monetary burden associated with the gout can be limited by promoting awareness, prior diagnosis, life style modification and through optimizing treatment strategies [18].

\section{Epidemiology}

Globally, the most common type of inflammatory arthritis that prevailed in human population is gout [19]. The epidemiological data suggests that the rate of incidence and prevalence of gout has tremendously increased since last few years. In United States (US), and Europe the prevalence of gout has increased many folds since last twenty years [13]. In United Kingdom (UK), more than 700,000 people are suffering from gout. It has also been estimated that annually about four million outdoor gout patients visit hospitals in United Kingdom [20]. According to another estimate, out of every thousand patients, 1.4 females and 4 males are affected by the gouty arthritis every year [21]. Studies suggest that disease is more prevalent among males than females especially in those who are above forty five years of age. On contrary, the risk of incidence of hyperuricemia increases many times in post-menopausal females. Incidence rate of gout and hyperuricemia is quite high in America too where around 3 million self-reported cases were documented in a survey [22]. Monetary burden associated with gout is believed to increase in United Kingdom due to suboptimal treatment and management of the condition [23]. In New Zealand, around 14.9\% of pacific island men and about $9.3 \%$ to $13.9 \%$ of Māori men are affected by the severe gout [24]. Higher number of hospital visits due to gout is reported in Asian than Caucasian [25]. Frequency of gout is also seem to increase in mainland of China [26] while in Germany, more than $1 \%$ of adult population is found to be effected by this disease [27].

\section{Pathophysiology}

\subsection{Production of urate crystals}

Gout is a metabolic disorder that arises due to increase formation of uric acid [28]. Uric acid is an end product of purine metabolism. In body, purines are converted to the hypoxanthine which is then converted into the uric acid by the action of hypoxanthine oxidase. In mammals, uric acid is converted into the allantoin by the action of an enzyme named uricase which is readily excreted by the kidneys [29]. Underlying mechanism for the development of gout is the increased serum level of uric acid due to decrease renal excretion [30]. Excretion of uric acid is largely determined by the renal reabsorption and secretion. The urate transporter 1 is involved in reabsorption process of uric acid. The increased formation and decreased excretion cause an increase in the serum concentration of uric acid which is subsequently converted to the monosodium urate crystals [31].

\subsection{Inflammatory response in gout}

The uric acid exists as needle shape crystals which are identified and ingested by the monocytes and neutrophils. Release of interleukin-1 and other cytokines initiate the inflammatory response which subsequently triggers the acute gout attack. Neutrophils with ingested crystals get closed and tightly packed with each other. Consequently, progress to the cell death following a unique pattern which is called tophaceous gout [32]. Inflammosome comprising of multimolecular structure is a pro-cytokine that activates the interleukin-1 which triggers the inflammatory response. Other mediators that are involved in inflammation include alpha-tissue necrosis factor and interleukin-6 [5]. The interleukin1 inhibitors block the discharge of interleukin-1 and thus assist to subside the inflammatory response [33]. 


\section{Signs and symptoms}

Acute gout is presented as intense pain, swelling and redness around the joints. Inter-critical gout is the term that refers to the asymptomatic period between the gout attacks [34]. Acute gout can be presented with high fever, leukocytosis and shading skin around the inflamed area, which usually resembles to a condition called cellulitis [35]. The term "podegra" is specified to acute gout when first metatarsophalangeal joints are affected by the urate crystals [36]. In acute gout, flares of distinctive pattern are also produced which are presented as the highly inflamed area and exhibited with pain [37] which usually last for about 5-10 days [38]. Asymptomatic hyperuricemia can persist for many years and in this period flares can be resolved. Whereas during intervals, crystals can propagate and pile up in joints and may cause pain, inflammation and destruction of joints which finally results into chronic gouty condition [39]. In chronic gout, tophi are produced that have unique features and can be diagnosed by the physical and imaging examination. These tophi appear at the various regions i.e. bone, cutaneous tissues and articular spaces [40]. Due to these flares, patients with chronic gout experience difficulty in their daily social activities and in addition to this, restricted mobility also negatively impacts their productivity [41]. Urate crystals may also deposit in different areas of eye which is usually asymptomatic. Ulcerative keratitis may occur if crystals stacked together in cornea but this is a rare condition [42].

\section{Diagnosis}

Diagnosis is usually based on clinical judgment although identification of urate crystals is of gold standard but it is seldom used in routine practice [43]. When synovial aspiration in routine cannot be performed then clinical diagnosis is usually preferred which is based on physical examination and history of patient [44]. Dual energy computed tomography (CT) is noninvasive and sensitive technique used to detect uric acid crystals [45]. It produces colored images of crystals and is helpful in identifying the subclinical tophus and tophus volume [46]. Other methods for detection of crystals include ultrasonography and microscopy [47].

\section{Prevention}

Monetary burden for the treatment of gout has increased significantly hence it calls for the need of its prevention by identifying the risk factors and modification of lifestyle [48]. Increased intake of coffee, soft drinks and fructose boost the risk of gout. Proper dietary recommendations with lifestyle modification can help to prevent the gout [49]. Protective measures for gout include decreased consumption of animal protein diet, drinking more fluid and increased the $\mathrm{pH}$ of urine [50]. Consumption of vegetables, nuts, fruits, whole grains, dairy products, vitamin $\mathrm{C}$ supplements with weight reduction and exercise can also curtail the risk of gout [51].

\section{Treatment}

Management and treatment of gout is based on decreasing the serum level of uric acid below $6 \mathrm{mg} / \mathrm{dl}$ with the help of medicines such as allopurinol and probenecid [52]. Customary medications for the treatment include colchicine, nonsteroidal anti-inflammatory drugs (NSAIDs) and adrenocorticotropin hormone [53]. Xanthine oxidase inhibitors in combination with uricosuric agents are also commonly used [54]. Systemic corticosteroids are effective in management of acute gout when used for short term doesn't produce no significant adverse effects [55]. Those patients who are resistant to or are contraindicated to allopurinol therapy can be managed by febuxostat which is an alternate drug for the treatment of gout [56].

\section{Conclusion}

Despite well identified risk factors and clear pathophysiology, gout still remains a major health concern. To cut off the global health burden and to improve the patient's Qol there is an utmost need: to reduce exposure to risk factors, to promote awareness, to institute robust prophylactic measures, to modify the lifestyle, to establish signs for early diagnosis and to develop optimized treatment strategies and policies. 


\section{Compliance with ethical standards}

\section{Acknowledgments}

Authors would like to acknowledge University College of Pharmacy, University of the Punjab, Lahore, Pakistan and Lahore Pharmacy College, Lahore Medical and Dental College, Lahore, Pakistan for their kind support.

\section{Disclosure of conflict of interest}

There is no conflict of interest among authors.

\section{References}

[1] R Buckley H. (2011). Epidemiology of gout: perspectives from the past.Current Rheumatology Reviews, 7 (2), 106-113.

[2] Doherty M, Jansen TL, Nuki G, Pascual E, Perez-Ruiz F, Punzi L, So AK and Bardin T. (2012). Gout: why is this curable disease so seldom cured? Annals of the rheumatic diseases, annrheumdis-2012-201687.

[3] Hasturk AE, Basmaci M, Canbay S, Vural C and Erten F. (2012). Spinal gout tophus: a very rare cause of radiculopathy. European Spine Journal, 21 (4), 400-403.

[4] Choi HK, Willett W and Curhan G. (2010). Fructose-rich beverages and risk of gout in women. Jama, 304 (20), 2270-2278.

[5] Abeles AM, Park JY, Pillinger MH and Cronstein BN. (2007). Update on gout: Pathophysiology and potential treatments. Current pain and headache reports, 11 (6), 440-446.

[6] Keenan RT, O'Brien WR, Lee KH, Crittenden DB, Fisher MC, Goldfarb DS, Krasnokutsky S, Oh C and Pillinger MH. (2011). Prevalence of contraindications and prescription of pharmacologic therapies for gout. The American journal of medicine, 124 (2), 155-163.

[7] Choi HK and Curhan G. (2007). Independent impact of gout on mortality and risk for coronary heart disease. Circulation, 116 (8), 894-900.

[8] Terkeltaub R. (2010). Update on gout: new therapeutic strategies and options. Nature Reviews Rheumatology, 6 (1), 30-38.

[9] Edwards NL. (2008). The role of hyperuricemia and gout in kidney and cardiovascular disease. Cleveland Clinic journal of medicine, 75 S13-6.

[10] Chilappa CS, Aronow WS, Shapiro D, Sperber K, Patel U and Ash JY. (2010). Gout and hyperuricemia. Comprehensive therapy, 36 3-13.

[11] Seminog 00 and Goldacre MJ. (2013). Gout as a risk factor for myocardial infarction and stroke in England: evidence from record linkage studies. Rheumatology, 52 (12), 2251-2259.

[12] Perez-Ruiz F and Lioté F. (2007). Lowering serum uric acid levels: what is the optimal target for improving clinical outcomes in gout? Arthritis Care \& Research, 57 (7), 1324-1328.

[13] Zhou L, Liu L, Liu X, Chen P, Zhang Y, Wu Y, Pettigrew JC, Cheng D and Yi D. (2014). Systematic review and metaanalysis of the clinical efficacy and adverse effects of Chinese herbal decoction for the treatment of gout. PLoS One, 9 (1), e85008.

[14] Smith E, Hoy D, Cross M, Merriman TR, Vos T, Buchbinder R, Woolf A and March L. (2014). The global burden of gout: estimates from the Global Burden of Disease 2010 study. Annals of the rheumatic diseases, 73 (8), 14701476.

[15] Dalbeth N, Petrie KJ, House M, Chong J, Leung W, Chegudi R, Horne A, Gamble G, McQueen FM and Taylor WJ. (2011). Illness perceptions in patients with gout and the relationship with progression of musculoskeletal disability. Arthritis Care \& Research, 63 (11), 1605-1612.

[16] Spencer K, Carr A and Doherty M. (2012). Patient and provider barriers to effective management of gout in general practice: a qualitative study. Annals of the rheumatic diseases, 71 (9), 1490-1495.

[17] Kiadaliri AA and Englund M. (2018). Temporal trends and regional disparity in rheumatoid arthritis and gout hospitalizations in Sweden, 1998-2015. Clinical Rheumatology, 1-6. 
[18] Wertheimer A, Morlock R and Becker MA. (2013). A revised estimate of the burden of illness of gout. Current Therapeutic Research, 75 1-4.

[19] Simeunovic Ostojic M and Maas J. (2018). Anorexia nervosa and uric acid beyond gout: An idea worth researching. International Journal of Eating Disorders,

[20] Dehghan A, Köttgen A, Yang Q, Hwang S-J, Kao WL, Rivadeneira F, Boerwinkle E, Levy D, Hofman A and Astor BC. (2008). Association of three genetic loci with uric acid concentration and risk of gout: a genome-wide association study. The Lancet, 372 (9654), 1953-1961.

[21] Roddy E and Doherty M. (2010).Gout. Epidemiology of gout.Arthritis research \& therapy, 12 (6), 223.

[22] Lawrence RC, Felson DT, Helmick CG, Arnold LM, Choi H, Deyo RA, Gabriel S, Hirsch R, Hochberg MC and Hunder GG. (2008). Estimates of the prevalence of arthritis and other rheumatic conditions in the United States: Part II. Arthritis \& Rheumatology, 58 (1), 26-35.

[23] Kuo C-F, Grainge MJ, Mallen C, Zhang W and Doherty M. (2014). Rising burden of gout in the UK but continuing suboptimal management: a nationwide population study. Annals of the rheumatic diseases, annrheumdis-2013204463.

[24] Hollis-Moffatt JE, Xu X, Dalbeth N, Merriman ME, Topless R, Waddell C, Gow PJ, Harrison AA, Highton J and Jones PB. (2009). Role of the urate transporter SLC2A9 gene in susceptibility to gout in New Zealand Māori, Pacific Island, and Caucasian case-control sample sets. Arthritis \& Rheumatology, 60 (11), 3485-3492.

[25] Krishnan E, Lienesch D and Kwoh CK. (2008). Gout in ambulatory care settings in the United States. The Journal of rheumatology, 35 (3), 498-501.

[26] Liu R, Han C, Wu D, Xia X, Gu J, Guan H, Shan Z and Teng W. (2015). Prevalence of hyperuricemia and gout in mainland China from 2000 to 2014: A systematic review and meta-analysis. BioMed research international, 2015

[27] Khanna PP, Nuki G, Bardin T, Tausche A-K, Forsythe A, Goren A, Vietri J and Khanna D. (2012). Tophi and frequent gout flares are associated with impairments to quality of life, productivity, and increased healthcare resource use: results from a cross-sectional survey. Health and quality of life outcomes, 10 (1), 117.

[28] So AK and Martinon F. (2017). Inflammation in gout: mechanisms and therapeutic targets. Nature Reviews Rheumatology, 13 (11), 639.

[29] Maiuolo J, Oppedisano F, Gratteri S, Muscoli C and Mollace V. (2016). Regulation of uric acid metabolism and excretion. International journal of cardiology, 213 8-14.

[30] Taniguchi A and Kamatani N. (2008). Control of renal uric acid excretion and gout. Current opinion in rheumatology, 20 (2), 192-197.

[31] Eraly SA, Vallon V, Rieg T, Gangoiti JA, Wikoff WR, Siuzdak G, Barshop BA and Nigam SK. (2008). Multiple organic anion transporters contribute to net renal excretion of uric acid. Physiological genomics, 33 (2), 180-192.

[32] Schett G, Schauer C, Hoffmann M and Herrmann M. (2015). Why does the gout attack stop? A roadmap for the immune pathogenesis of gout. RMD open, 1 (Suppl 1), e000046.

[33] So A. (2007). Recent advances in the pathophysiology of hyperuricemia and gout. Revue medicale suisse, 3 (103), $720,722-4$.

[34] Seth R, Kydd A, Buchbinder R, Bombardier C and Edwards CJ. (2013). Allopurinol for chronic gout. Cochrane Database Syst Rev, 4

[35] Eggebeen AT. (2007). Gout: an update. American family physician, 76 (6),

[36] Busso N and So A. (2010).Gout. Mechanisms of inflammation in gout.Arthritis research \& therapy, 12 (2), 206.

[37] Khanna D, Fitzgerald JD, Khanna PP, Bae S, Singh MK, Neogi T, Pillinger MH, Merill J, Lee S and Prakash S. (2012). 2012 American College of Rheumatology guidelines for management of gout. Part 1: systematic nonpharmacologic and pharmacologic therapeutic approaches to hyperuricemia. Arthritis Care \& Research, 64 (10), 1431-1446.

[38] Doghramji PP and Wortmann RL. (2012). Hyperuricemia and gout: new concepts in diagnosis and management. Postgraduate medicine, 124 (6), 98-109.

[39] Mandell BF. (2008). Clinical manifestations of hyperuricemia and gout. Cleveland Clinic journal of medicine, 75 S5-8. 
[40] Dalbeth N, Aati O, Gao A, House M, Liu Q, Horne A, Doyle A and McQueen FM. (2012). Assessment of tophus size: a comparison between physical measurement methods and dual-energy computed tomography scanning. JCR: Journal of Clinical Rheumatology, 18 (1), 23-27.

[41] Edwards N, Sundy J, Forsythe A, Blume S, Pan F and Becker M. (2011). Work productivity loss due to flares in patients with chronic gout refractory to conventional therapy. Journal of medical economics, 14 (1), 10-15.

[42] Yazdanyar A, Rizzuti AE, Mechel E, Denisova K and Lazzaro DR. (2018). Gout keratitis: a case of peripheral ulcerative keratitis secondary to gout with a review of the literature. Cornea, 37 (3), 379-381.

[43] Peláez-Ballestas I, Cuevas CH, Burgos-Vargas R, Roque LH, Terán L, Espinoza J, Esquivel-Valerio JA, GoycocheaRobles MV, Aceves FJ and Bernard AG. (2010). Diagnosis of chronic gout: evaluating the american college of rheumatology proposal, European league against rheumatism recommendations, and clinical judgment. The Journal of rheumatology, 37 (8), 1743-1748.

[44] Dore RK. (2008). The gout diagnosis. Cleveland Clinic journal of medicine, 75 S17-21.

[45] Glazebrook KN, Guimarães LS, Murthy NS, Black DF, Bongartz T, J. Manek N, Leng S, Fletcher JG and McCollough CH. (2011). Identification of intraarticular and periarticular uric acid crystals with dual-energy CT: initial evaluation. Radiology, 261 (2), 516-524.

[46] Choi HK, Al-Arfaj AM, Eftekhari A, Munk PL, Shojania K, Reid G and Nicolaou S. (2009). Dual energy computed tomography in tophaceous gout. Annals of the rheumatic diseases, 68 (10), 1609-1612.

[47] Perez-Ruiz F, Dalbeth N and Bardin T. (2015). A review of uric acid, crystal deposition disease, and gout. Advances in therapy, 32 (1), 31-41.

[48] Choi HK and Curhan G. (2008). Soft drinks, fructose consumption, and the risk of gout in men: prospective cohort study. Bmj, 336 (7639), 309-312.

[49] Hak AE and Choi HK. (2008). Lifestyle and gout. Current opinion in rheumatology, 20 (2), 179-186.

[50] Liebman SE, Taylor JG and Bushinsky DA. (2007). Uric acid nephrolithiasis. Current rheumatology reports, 9 (3), 251-257.

[51] Choi HK. (2010). A prescription for lifestyle change in patients with hyperuricemia and gout. Current opinion in rheumatology, 22 (2), 165-172.

[52] Sundy JS and Hershfield MS. (2007). Uricase and other novel agents for the management of patients with treatment-failure gout. Current rheumatology reports, 9 (3), 258-264.

[53] Sivera F, Wechalekar MD, Andrés M, Buchbinder R and Carmona L. (2012). Interleukin-1 inhibitors for acute gout. The Cochrane database of systematic reviews, 9

[54] Diaz-Torné C, Perez-Herrero N and Perez-Ruiz F. (2015). New medications in development for the treatment of hyperuricemia of gout. Current opinion in rheumatology, 27 (2), 164-169.

[55] Janssens HJ, Lucassen PL, Van de Laar FA, Janssen M and Van de Lisdonk EH. (2008). Systemic corticosteroids for acute gout. The Cochrane Library,

[56] Stevenson M and Pandor A. (2011). Febuxostat for the management of hyperuricaemia in patients with gout. Pharmacoeconomics, 29 (2), 133-140.

\section{How to cite this article}

Ashiq K, Latif A, Ashiq S, Sundus A. (2018). A systematic review on the prevalence, pathophysiology, diagnosis, management and treatment of gout (2007-2018). GSC Biological and Pharmaceutical Sciences, 5(1), 50-55. 